\title{
USO E CONSERVAÇÃO DE EQUIPAMENTOS DE PROTEÇÃO INDIVIDUAL (EPIS) EM UMA UNIDADE DE ALIMENTAÇÃO E NUTRIÇÃO HOSPITALAR
}

\section{USE AND CONSERVATION OF PERSONAL PROTECTIVE EQUIPMENT IN A HOSPITAL FOOD AND NUTRITION UNIT}

\author{
Alessandra Varanda Picchi ${ }^{1}$ \\ Andrea Carvalheiro Guerra Matias ${ }^{2}$ \\ Mônica Glória Neumann Spinelli ${ }^{3}$
}

\begin{abstract}
Resumo: No Brasil, o setor de alimentação institucional é um mercado em expansão, mas ainda se observam condições de trabalho impróprias. O objetivo deste estudo foi levantar as condições de segurança e avaliar a utilização de Equipamentos de Proteção Individual (EPIs) por parte dos colaboradores de uma Unidade de Alimentação e Nutrição Hospitalar (UAN Hospitalar), de um hospital privado do município de São Paulo. Realizou-se um levantamento junto aos funcionários para verificação de EPIs, disponibilidade e conservação, previstos no Manual de Boas Práticas. A unidade apresentou todos os EPIs previstos em boas condições de conservação. Observou-se $83 \%$ de frequência do uso dos EPIs pelos colaboradores. As áreas ou atividades que apresentaram diferentes graus de inadequação, onde um ou mais colaboradores não faziam uso de EPI, foram câmaras frias e refrigeradas, o manuseio de facas, higienização das coifas, preparo do café, corte de carnes, lavagem de panelas, uso do processador de alimentos, corte de pães e uso da fritadeira. Apesar de ser observada uma frequência de adequação de uso de $83 \%$, fazem-se necessários o treinamento e motivação continuados, bem como a sistematização do controle do correto uso de EPIs, a fim de garantir a segurança dos colaboradores.
\end{abstract}

Palavras chave: Segurança no trabalho; equipamentos de proteção; restaurantes.

Abstract: In Brazil, the institutional food service sector is a booming market, but it is still possible to observe inappropriate work conditions in Food and Nutrition Units. The present study aimed to perform a survey on safety assessment and use of Personal Protective Equipment (PPE) by the employees of a private Hospital Food and Nutrition Unit, in the city of São Paulo. The survey was conducted among the employees for PPE verification (availability and conservation) as foreseen in the Good Practices Manual. The unit had all PPE provided in good conditions. It was observed that $83 \%$ of employees make the appropriate use of PPE. The areas or activities that showed different degrees of inadequacy, where one or more employees did not use PPE, were cold rooms and cold storage, handling knives, cleaning of exhaust hoods, preparation of coffee, meat cutting, washing pots, usage of food processor, bread slicing and use of the fryer. Despite the frequency of usability observed (83\%), continuous training and motivating, as well as the systematic control of the correct use of PPE, are necessary in order to ensure the safety of the employees.

Keywords: Occupational health; protective devices; restaurants.

\section{INTRODUÇÃO}

Ao longo da história, mortes, doenças e mutilações de trabalhadores estiveram frequentemente relacionadas ao ambiente de trabalho. As atividades laborais, desde

\footnotetext{
${ }^{1}$ Nutricionista formada pela Universidade Presbiteriana Mackenzie, Brasil. E-mail: lele.picchi@hotmail.com. ${ }^{2}$ Nutricionista, professora adjunta do curso de nutrição da Universidade Presbiteriana Mackenzie, Brasil. Email: acgmatias@gmail.com.

${ }^{3}$ Nutricionista, professora adjunta do curso de nutrição da Universidade Presbiteriana Mackenzie, Brasil. Email: spinelli@mackenzie.br.
} 
épocas remotas, sempre apresentaram riscos em potencial (MELO JÚNIOR e RODRIGUES, 2005).

As doenças ocupacionais, profissionais, ou do trabalho resultam da exposição aos riscos ambientais, ergonômicos ou acidentes, sendo caracterizadas sempre que se estabeleça o nexo causal entre determinados riscos ocupacionais e os danos observados na saúde do trabalhador (CONCEIÇÃO; CAVALCANTI, 2001).

Segundo Abreu e Spinelli (2016), dados oficiais de diversos estudos apontam que a morbimortalidade de trabalhadores brasileiros teve um aumento significativo ao longo dos anos, mostrando a necessidade de buscar meios de promoção de condições seguras e saudáveis aos colaboradores. De modo geral, essa preocupação com a saúde do colaborador da Unidade de Alimentação e Nutrição (UAN), surge à medida da conscientização de que as condições de trabalho e saúde estão intrinsecamente relacionadas com a performance e a produtividade (ANTUNES; SPINELLI; KOGA, 2005).

Conforme a Norma Regulamentadora no6 (NR-6), do Ministério do Trabalho, o Equipamento de Proteção Individual (EPI) é considerado todo dispositivo ou produto de uso individual utilizado pelos trabalhadores, determinado a proteger de riscos suscetíveis que possam ameaçar a segurança e a saúde no trabalho. Por meio dessa norma, tornou-se obrigatório que toda empresa forneça, gratuitamente, EPIs adequados ao risco, em perfeito estado de conservação e funcionamento, a todos os colaboradores e que se faça a substituição imediata caso estes estejam danificados ou tenham sido extraviados, além de cumprir e fazer cumprir as normas de segurança oficiais (BRASIL, 2001).

Cabe ao trabalhador ter conhecimento sobre as referidas normas de segurança e fazer uso, obrigatório, dos EPIs sempre que a atribuição designada ofereça possíveis riscos à sua integridade física. É necessário garantir a conservação e, ainda, a imprescindível comunicação à Segurança do Trabalho sempre que os EPIs apresentem alterações ou danos em sua funcionalidade (BRASIL, 2001).

No Brasil, o setor de alimentação institucional tem sido um mercado em expansão. Atualmente, segundo a Associação Brasileira das Empresas de Refeições Coletivas (ABERC, 2017), este setor emprega 180 mil colaboradores. Apesar disso, ainda é possível observar condições de trabalho impróprias em UANs, como a ergonomia de áreas ocupacionais, que levam a insatisfação e cansaço excessivo aos funcionários e, com isso, a queda na produtividade e problemas ligados à saúde e acidentes funcionais (ABREU; SPINELLI, 2016).

Todos os setores da UAN apresentam, embora em diferentes graus, riscos em potencial, que podem ser advindos da atividade ocupacional ou do ambiente (CONTRI; JAPUR; VIEIRA, 2015; ABREU; SPINELLI, 2016). 
O presente estudo apresentou como objetivo a realização de um levantamento das condições de segurança e avaliação da utilização de EPls por parte dos colaboradores em uma UAN Hospitalar após treinamento.

\section{MÉTODO}

Foi conduzido um estudo de caráter transversal, com coleta de dados primários, com os 18 funcionários de uma UAN Hospitalar, de um hospital privado do município de São Paulo, para levantamento da prevalência de uso de EPIs, a partir das normas descritas no Manual de Boas Práticas da unidade, para verificação de disponibilidade e de estado de conservação.

O colaborador recebe treinamento na admissão e capacitações periódicas. Após capacitação sobre segurança no trabalho e uso dos EPIs, realizado pela técnica em nutrição da unidade, foi aplicado um formulário observacional baseado na norma regulamentadora NR6 - Programa de Controle Médico de Segurança Ocupacional. As Normas Regulamentadoras da Secretaria de Segurança no Trabalho (2011) são instrumentos adaptados por Abreu, Spinelli e Souza Pinto, 2011, para a avaliação da conformidade do uso de EPIs, de acordo com cada área de trabalho e a função do colaborador.

O projeto obteve aprovação segundo CIEP N001/02/11 e foi desenvolvido no primeiro semestre de 2012.

O instrumento foi desenvolvido levando em consideração as áreas e as atividades nas quais se faz necessário o uso de EPIs, descritos no Manual de Boas Práticas da unidade, totalizando 20 itens elencados, durante quatro dias não consecutivos, no total de duas semanas (8 observações). Todos os EPIs estavam em boas condições de conservação, em número suficiente de acordo com as normas regulamentadoras (BRASIL, 2001).

Para consideração do item adequação foi utilizada a frequência de utilização do EPI em cada área de trabalho ou atividade, durante o período de observação. Para o cômputo da frequência, o funcionário deveria utilizar todos os EPIs esperados para a atividade e/ou área, conforme o Manual de Boas Práticas da unidade e descritos no Quadro 1.

Quadro 1 - EPIs disponíveis segundo a área de trabalho e atividades. São Paulo, 2012.

\begin{tabular}{|c|l|}
\hline EPI & \multicolumn{1}{c|}{ Área de trabalho/ atividades } \\
\hline Óculos de segurança & $\begin{array}{l}\text { Lavagem de coifas, utilização de produtos químicos, frituras, } \\
\text { lavagens em geral. }\end{array}$ \\
\hline
\end{tabular}




\begin{tabular}{|l|l|}
\hline Protetor auricular & $\begin{array}{l}\text { Devolução de bandejas, conforme ultrapassar limite de } \\
\text { tolerância no PPRA. }\end{array}$ \\
\hline Mangote de lonita & Cocção e frituras. \\
\hline Luvas de PVC & $\begin{array}{l}\text { Lavagem de bandejas, limpeza em geral, devolução e } \\
\text { atividades com produtos químicos. }\end{array}$ \\
\hline Luvas de PVC cano longo & $\begin{array}{l}\text { Lavagem em geral, higienização de panelas, atividade com } \\
\text { produtos químicos. }\end{array}$ \\
\hline Luva térmica & Cocção, fornos, frituras e assados. \\
\hline Luva de malha de aço & Açougue, salada, confeitaria, quaisquer atividades com cortes. \\
\hline Avental de PVC & Salada, limpeza e higienização em geral. \\
\hline Avental térmico & Cocção, fornos, frituras e assados. \\
\hline Casaco térmico & Câmaras refrigeradas. \\
\hline $\begin{array}{l}\text { Conjunto térmico completo } \\
\text { (blusão, luvas, calças e } \\
\text { botas PVC forradas) e } \\
\text { perneira }\end{array}$ & Atividade em câmaras frias (abaixo de $0^{\circ}$ C).. \\
\hline Calçado de segurança & Demais áreas da cozinha e restaurante. \\
\hline Botas de PVC & Higienização na cozinha em geral/ áreas encharcadas. \\
\hline
\end{tabular}

Para avaliação dos dados foi aplicada classificação segundo exigências internas na unidade de alimentação e nutrição do hospital, sendo "Ótimo", quando a frequência de uso dos EPIs foi superior ou igual a 90\%, "Bom", entre 75 e 89\%, "Regular", quando entre 60 a $74 \%$ e "Ruim", quando inferior a $60 \%$.

Os dados foram tabulados no Microsoft Office Excel 2007 sendo as variáveis qualitativas apresentadas segundo frequência absoluta e relativa.

\section{RESULTADOS E DISCUSSÃO}

Foram encontrados, na UAN Hospitalar, todos os EPIs previstos no Manual de Boas Práticas da unidade e em número suficiente para todas as áreas e atividades.

Todos os EPIs apresentaram bom estado de conservação e condições de uso. De acordo com a NR-6, cabe ao empregador, orientar, treinar e exigir do trabalhador o uso adequado, guarda e conservação dos EPIs e cabem aos funcionários cumprirem as determinações do empregador sobre o uso adequado, guarda e conservação desses instrumentos (BRASIL, 2001).

No total de observações, verificou-se $83 \%$ de adequação de utilização dos EPIs pelos colaboradores da unidade, após a capacitação periódica sobre segurança no trabalho e uso de EPIs, o que corresponde a classificação de "bom" segundo as exigências internas da unidade (Tabela 1). 
Tabela 1 - Frequência de utilização de EPls segundo área/atividade. São Paulo, 2012.

\begin{tabular}{|c|c|c|c|c|c|c|c|c|c|c|}
\hline \multirow{3}{*}{$\begin{array}{l}\text { Equipamentos/ } \\
\text { Atividades }\end{array}$} & \multicolumn{10}{|c|}{ Utilização de EPIs } \\
\hline & \multicolumn{2}{|c|}{$1^{\circ} \mathrm{dia}$} & \multicolumn{2}{|c|}{$2^{\circ} \mathrm{dia}$} & \multicolumn{2}{|c|}{$3^{\circ} \mathrm{dia}$} & \multicolumn{2}{|c|}{$4^{\circ} \mathrm{dia}$} & \multicolumn{2}{|c|}{ total } \\
\hline & $n$ & $\%$ & $\mathrm{n}$ & $\%$ & $\mathrm{n}$ & $\%$ & $\mathrm{n}$ & $\%$ & $\mathrm{n}$ & $\%$ \\
\hline $\begin{array}{l}\text { Câmaras Refrigeradas } \\
\text { e frias }\end{array}$ & 3 & 75 & 3 & 75 & 3 & 75 & 1 & 25 & 10 & 63 \\
\hline Transporte manual & 4 & 100 & 4 & 100 & 4 & 100 & 4 & 100 & 16 & 100 \\
\hline $\begin{array}{l}\text { Descascador de } \\
\text { legumes }\end{array}$ & 4 & 100 & 4 & 100 & 4 & 100 & 4 & 100 & 16 & 100 \\
\hline $\begin{array}{l}\text { Higienização } \\
\text { instalações }\end{array}$ & 4 & 100 & 3 & 75 & 4 & 100 & 4 & 100 & 15 & 94 \\
\hline Manuseio de facas & 3 & 75 & 2 & 67 & 2 & 67 & 2 & 67 & 9 & 69 \\
\hline Higienização de coifas & 3 & 75 & 2 & 50 & 3 & 75 & 3 & 75 & 11 & 69 \\
\hline Preparo do café & 2 & 50 & 2 & 50 & 2 & 50 & 2 & 50 & 8 & 50 \\
\hline Corte de carnes & 3 & 75 & 3 & 75 & 3 & 75 & 3 & 75 & 12 & 75 \\
\hline Lavar louças & 3 & 60 & 3 & 67 & 1 & 20 & 3 & 60 & 10 & 52 \\
\hline Picador manual & 2 & 100 & 2 & 100 & 2 & 100 & 2 & 100 & 8 & 100 \\
\hline Caldeirão a vapor & 3 & 100 & 3 & 100 & 2 & 67 & 3 & 100 & 11 & 92 \\
\hline $\begin{array}{l}\text { Processador de } \\
\text { alimentos }\end{array}$ & 2 & 67 & 2 & 67 & 2 & 67 & 2 & 50 & 8 & 63 \\
\hline Liquidificador & 2 & 100 & 2 & 100 & 2 & 100 & 2 & 100 & 8 & 100 \\
\hline $\begin{array}{l}\text { Manuseio de utensílios } \\
\text { quentes }\end{array}$ & 3 & 100 & 3 & 100 & 3 & 100 & 3 & 100 & 12 & 100 \\
\hline $\begin{array}{l}\text { Manuseio e transporte } \\
\text { de alimentos }\end{array}$ & 2 & 100 & 2 & 100 & 2 & 100 & 2 & 100 & 8 & 100 \\
\hline Chapa/ Grelha & 3 & 100 & 3 & 100 & 3 & 100 & 2 & 67 & 11 & 92 \\
\hline Corte de pães & 2 & 67 & 2 & 67 & 2 & 67 & 2 & 67 & 8 & 67 \\
\hline Batedeira & 2 & 100 & 2 & 100 & 2 & 100 & 2 & 100 & 8 & 100 \\
\hline Fritadeira & 5 & 100 & 3 & 60 & 4 & 80 & 3 & 60 & 15 & 75 \\
\hline Descarregar alimentos & 2 & 100 & 2 & 100 & 2 & 100 & 2 & 100 & 8 & 100 \\
\hline Total & 57 & 87 & 52 & 83 & 52 & 82 & 51 & 80 & 212 & 83 \\
\hline
\end{tabular}

A prevalência de uso dos EPIs foi classificada como "Ótimo" para as áreas/ atividades a seguir: transporte manual de cargas, descascamento de legumes, higienização das instalações, utilização do picador manual, caldeirão a vapor, chapa, batedeira e liquidificador, manuseio de utensílios quentes, manuseio e transporte de alimentos para pacientes, descarregamento de alimentos. 
As áreas e/ou atividades que apresentaram maiores graus de inadequação, com classificação "regular" foram as Câmaras refrigeradas e frias, Manuseio de facas, Higienização de coifas, Processador de alimentos, Corte de pães, e classificação "ruim" o Preparo de café e Lavar louças. Nestes casos um ou mais colaboradores não faziam uso de EPI.

No caso das câmaras frias e refrigeradas, a não utilização do casaco térmico pode ocasionar problemas de saúde aos funcionários por variações térmicas decorrentes da alternância com a cozinha, que é um ambiente que geralmente apresenta alta temperatura. Segundo Beck (2008), a passagem de um ambiente refrigerado para outro aquecido, ou vice-versa, pode ocasionar choque térmico ao organismo do indivíduo e comprometer sua defesa imunológica, tornando-o suscetível a vírus e bactérias, e provocar doenças respiratórias. Estudo de Schwab et al. (2005) mostrou que, entre os EPI utilizados, um dos que oferecem maior resistência por parte dos funcionários, quanto à sua utilização, é o casaco térmico, que os funcionários relataram utilizar apenas quando realizada a limpeza e organização da câmara fria, atividade em que a permanência na câmara é maior.

Foi observado na prática que o não uso do casaco térmico se deve à pressão temporal do serviço aliado ao número de vezes que os funcionários adentram nas câmaras, e até mesmo ao fato da falta de higienização frequente do EPI, o que torna desagradável seu uso.

Verificou-se que no manuseio de equipamentos relacionados ao risco de acidentes por meio de cortes, tais como no uso de facas (corte de carnes, pães), processador de alimentos, bem como na chapa e/ou grelha, o não uso de EPIs foi frequente por pelo menos um colaborador por dia. Na prática, muitas vezes, o não uso de EPIs resulta da inadequação do tamanho da luva à mão do funcionário e até mesmo à dificuldade de higienização posterior ao uso.

Observa-se que o mero conhecimento das medidas de prevenção e a formação centrada em aspectos técnicos podem não corresponder a uma maior adesão, na prática, a essas medidas, sendo que o gerenciamento dos riscos ligados a perfurocortantes requer a combinação de diferentes estratégias de intervenção, incluindo 0 uso de dispositivos de segurança (REINHARDT; FISCHER, 2009).

Foi observada baixa adesão ao uso de protetores auriculares. Para Abreu, Spinelli e Araújo (2002) é importante a exigência do uso desse equipamento e a realização de rodízio dos funcionários nas diversas atividades, além da supervisão do uso correto do equipamento.

Para evitar acidentes, a empresa deve oferecer, exigir e verificar o uso dos EPIs como luvas, e óculos no desempenho dessas atividades (CÂNDIDO; VIEIRA; 2003). É importante, ainda, considerar que o processo educativo, implicando em uma mudança de mentalidade, só obtém êxito em longo prazo (RODRIGUES,1998). 
Nas áreas de preparo de café, lavagem, e de cocção com o uso de fritadeira e caldeirão foi observado o descumprimento do uso de mangote e perneiras de PVC, equipamentos de extrema importância, que de acordo com a NR-6 (BRASIL, 2001) devem ser utilizados para a proteção dos braços e das pernas contra agentes cortantes e perfurantes, produtos químicos, agentes térmicos e para a proteção das pernas contra a umidade proveniente de operações com uso de água.

Segundo Oliveira (2003), com relação à prevenção de acidentes, a importância do comportamento do trabalhador, mesmo que tenha sido a causa preponderante, pode ter, no contexto geral, uma dimensão relativizada ou até mesmo secundária. $O$ que deve ser amplamente considerado e valorizado são os determinantes do comportamento, tais como problemas do ambiente e/ou das relações de trabalho e ainda da vida privada do trabalhador, que possam ter causado interferência no seu relacionamento com o todo de seu trabalho e definindo posturas que resultaram em atitudes corretas ou equivocadas.

Barbosa e Almeida (2008), em estudo sobre avaliação de riscos ambientais em uma UAN localizada na cidade de Botucatu, SP, relataram grande interesse dos colaboradores pelo treinamento voltado à segurança no trabalho, uma vez que tem aplicação direta no trabalho realizado, influenciando positivamente suas atividades. Isto mostra que o gestor da unidade não pode descuidar da capacitação da equipe. Segundo Ghisleni e Basso (2008), o treinamento deve visar não somente a multiplicação de conhecimentos, mas também a mudança de comportamento e de atitudes.

De acordo com Abreu e Spinelli (2011), cabe ao nutricionista, como administrador, identificar as áreas de risco ocupacional dentro da UAN e sua atuação frente às condições adversas, tendo em vista a importância do conhecimento e do controle dos riscos ocupacionais. Com estes dados mapeados, faz-se necessário instrumentar e educar os colaboradores para o seguimento dos requisitos de segurança. O treinamento é importante alternativa para a mudança de atitudes, conhecimentos ou habilidades necessárias ao desempenho adequado das atividades (LACERDA; ABBAD, 2003).

\section{CONCLUSÃO}

Apesar de a unidade ter realizado uma capacitação recente, apresentar todos os EPIs necessários, e em bom estado de conservação, nem sempre seu uso foi observado.

Para melhor assegurar a saúde e a qualidade de trabalho dos funcionários, fazse necessário aplicar treinamentos regulares, supervisão contínua e exigência do uso dos equipamentos de proteção, instrumentalizando os funcionários e criando uma conscientização de prevenção. 
É importante que o funcionário veja a utilização dos EPIs como fator de benefício e não de estorvo.

Faz-se relevante à academia científica, trabalhos desta natureza para ratificar a importância da capacitação e supervisão do uso de EPIs em unidades de alimentação e nutrição com vistas a garantir a segurança do trabalhador e produtividade do serviço.

\section{REFERÊNCIAS}

ABERC - ASSOCIAÇÃO BRASILEIRA DAS EMPRESAS DE REFEIÇÕES

COLETIVAS. Mercado real. Disponível em:

<http://www.aberc.com.br/mercadoreal.asp?IDMenu=21>. Acesso em: 24 dez. 2015.

ABREU, E.S; SPINELLI, M.G.N. Segurança e saúde no trabalho. In: ABREU, E.S; SPINELLI, M.G.N.; SOUZA PINTO, A.M. (col. Gestão de unidades de alimentação e nutrição: um modo de fazer. 4 ed. São Paulo: Metha, 2011.

ABREU, E.S.; SPINELLI, M.G.N.; ARAÚJO, R.M.V. Fatores de Risco Ambiental para trabalhadores de Unidade de Alimentação e Nutrição. Rev Nutr Pauta, São Paulo, v. 1, n. 57, p. 46-49, 2002.

ANTUNES, A.A; SPINELLI, M.G.N.; KOGA, R.A. Análise Ergonômica do Trabalho (AET) de uma Unidade de Alimentação e Nutrição (UAN) de São José dos Campos. Revista Nutrição em Pauta. São Paulo, p. 46-49, 2005.

BARBOSA, L.N., ALMEIDA, F.Q.A. Relato de experiência sobre a avaliação dos riscos ambientais e mapeamento em uma unidade de alimentação e nutrição (UAN) para a promoção da segurança no trabalho. Rev. Simbio-Logias, v.1, n.2, 2008.

BARROS, B.T; PRATES, M.A. O estilo brasileiro de administrar. São Paulo: Atlas, 1996.

BECK, A.C. U. Análise dos aspectos ergonômicos que envolvem a atividade profissional de camareira, auxiliar de cozinha e cozinheira no Hotel Jardim Europa em ljuí- RS. 2008, 82 p. Dissertação (Produção técnico científica para obtenção do título de bacharel em Turismo e Hotelaria) Universidade Vale do Itajaí. Balneário de Camboriú.

BRASIL. MINISTÉRIO DO TRABALHO E EMPREGO. NR-6 - Programa de Controle Médico de Segurança Ocupacional. Normas Regulamentadoras de da Secretaria de Segurança no Trabalho. 2001. Disponível em: <http://portal.mte.gov.br/data/files/FF8080812DC56F8F012DCDAD35721F50/NR06\%20 (atualizada)\%202010.pdf> Acesso em: 12 nov. 2012.

CANDIDO, I.; VIEIRA, E. Gestão de Hotéis: técnicas, operações e serviços. Caxias do Sul: Educs, 2003.

CONCEIÇÃO, M.L.C.; CAVALCANTI, C.L.C. Avaliação dos Equipamentos de Proteção Individual (EPIs) na Unidade de Alimentação e Nutrição (UAN) do Restaurante Universitário da UFPB. Rev. Conc., v. 4, n. 5, p. 1-12, 2001. 
CONTRI, P.V.; JAPUR, C.C.; VIEIRA, M.N.C.M. Saúde do trabalhador. In: VIEIRA, M.N.C.M; JAPUR, C.C. (coord.) Gestão da qualidade na produção de refeições. Rio de Janeiro: Guanabara Koogan, 2015.

GHISLENI, D. R.; BASSO, C.; Educação em saúde a manipuladores de duas Unidades de Alimentação e Nutrição do município de Santa Maria/Rs. Disc. Scientia.

Série: Ciências da Saúde, Santa Maria, v. 9, n. 1, p. 101-108, 2008.

LACERDA, E.R.M., ABBAD, G. Impacto do treinamento no trabalho: investigando variáveis motivacionais e organizacionais como suas preditoras. Rev Adm Contemp., v.7, n.4, Curitiba, 2003.

MELO JÚNIOR, A. S.; RODRIGUES, C. L. P. O risco de acidente de trabalho na indústria de panificação: o caso das máquinas de cilindro em massa. In: ENCONTRO NACIONAL DE ENGENHARIA DE PRODUÇÃO, 25, 2005, Porto Alegre. ENEGEP 2005. Disponível em: <http://www.segurancaetrabalho.com. br/download/indpanificacao.pdf>. Acesso em: 02 de out. 2012.

OLIVEIRA, J. C. de. Segurança e saúde no trabalho: uma questão mal compreendida. São Paulo Perspec., São Paulo, v. 17, n. 2, p. 03-12, June 2003. Disponível em: $<$ http://www.scielo.br/scielo.php?script=sci_arttext\&pid=S0102$88392003000200002 \& \mathrm{lng}=$ en\&nrm=iso $>$. Acesso em: 06 fev. 2018.

REINHARDT, E. L.; FISCHER, F. M. Barreiras às intervenções relacionadas à saúde do trabalhador do setor saúde no Brasil. Revista Panam Salud Publica, v. 25, n. 5, p. 411-7, 2009.

RODRIGUES, J.S. O moderno príncipe industrial: o pensamento pedagógico da Confederação Nacional da Indústria. Rio de Janeiro: Editores Autores Associados.1998, p. 82.

SCHWAB, J.T.G.; BARTH, E.; HERING, B.; LIMA, E.E.; LINKE, C. A importância da utilização de equipamentos de proteção individual por manipuladores de UAN. Nutrire, v. 30, n. Suplemento, p. 355-355, 2005. 